\section{PGT's IMIX-PTS}

PGT's IMIX-PTS - Position-Tagged Spectrometry has received the 1996 R\&D 100 Award as one of the year's most technologically significant new products. The award recognizes outstanding applied science developments and technological advances

What makes IMIX-PTS unique is the way X-ray data is collected. Position Tagged Spectrometry (PTS) detects $X$-rays, determines the $X$-ray energy, and encodes the photon with the sample $x, y$ coordinate While the electron beam rapidly scans the sample, all $X$-ray information is streamed to the computer with energy and position data for real time or subsequent post-analysis review.

Prior to PTS, the operator would begin the analysis by collecting an overall spectrum of the area of interest. If an element distribution map was desired, each element would be specified and a limited number collected during a single run. Should a missing element be discovered later, a separate map would be collected. Should areas of interest emerge from the maps and spectra be required from these regions, the operator would need to position the electron beam and acquire a separate specimen from each location. Finally, if a question was raised after the analysis, the sample would need to be returned to the electron microscope, the area in question found, and the analy. sis repeated

IMIX-PTS has been recognized as a breakthrough in EDS microanalysis because in one simple collection the analyst collects and archives all the data required from that sample: a high resolution electron image, an $X$-ray spectrum from the total scanned area, $X$-ray maps for all elements in the periodic table, and spectra from any selected regions of interest. Most significant is that a sample's stored image may be displayed in the future and a map for any element or an $X$-ray spectrum from any specilied region can be accessed and evaluated.

This new way of collecting and archiving $X$-ray data will benefit all microanalysis applications from rapid and efficient quality control through the most sophisticated research and development.

Princeton Gamma Tech, Inc. (609)924-7310, Fax: (609)924-1729. Circle Reader Inquiry \#40.

\section{Low-Cost Micromanipulation/Injection System}

The new economical Axiovert 25 inverted microscope from Carl Zeiss can be the platform of a low-cost micromanipulation and injection system for cell injection, cellular microsurgery and in vitro fertilization (IVF)

Two new stage mounting adapters, MNZ 13 and MNZ 131, allow the use of the full range of Narishige micromanipulators and positioners with the Axiovert 25. Another product available from Zeiss is the new Narishige G-100 Thin Wall Capillary Tube specifically designed for IVF and intra-cytoplasmic sperm injection (ICSI).

The Axiovert 25 offers outstanding stability and optical performance. It is the first inverted microscope that combines famous Zeiss ICS optics with the new VAREL variable relief contrast technique.

For further information. cpntact Carl Zeiss, Inc. Microscape Divisiion, One Zeiss Drive, Thornwood, NY 10594. (800)233-2343, Fax: (914)681-7446. Circle Reader Inquiry \#43

\section{CT3500 CRYO Transfer System Makes ultra-high resolution TEM work routine}

Oxford Instruments, Inc., Microanalysis Group's advanced CT 3500 Cryotransfer system allows frost-free transfer of both specimen and holder when used in conjunction with a Transmission Electron Microscope (TEM). It is both an ultra-stable low temperature sample holder and a cryo transfer workstation and guarantees a high stability performance with a resolution of $0.34 \mathrm{~nm}$ in all directions

With cryotransfer of TEM specimens, examination of frozen, hydrated or low melting point materials in the form of vitrified suspensions or cryo-sections with the potential for 3-D reconstruction of biological macromolecules by integrating images obtained at different orientations is possible. $X$-ray mapping of unfixed cryo sections allows the detection of ionic concentrations. And following sample preparation and coating, the CT3500 enables Cryo SEM to be performed in the STEM.

Other advantages of the sample cooling includes reduction in beam-damage, minimization of mass loss and associated TEM contamination, investigations of phases in materials only present below room temperature and improved crystallographic information.

Oxford Instruments, Microanalysis Group: (508)369-9933, Fax: (508)369-8287. Circle Reader Inquiry \#41.

\section{New LEICA EM KMR Balanced Break Glass Knifemaker}

Leica presents the high precision LEICA EM KMR balanced break glass knifemaker, featuring a new braking mechanism, simple alignment and ergonomic design.

The LEICA EM KMR knifemaker helps improve the quality and reproducibility of your knives. With the LEICA EM KMR knifemaker you can produce optimal knives for semi-thin light microscopy sectioning or for cutting ultrathin sections for ultramicrotomy and cryo. ultramicrotomy

The LEICA EM KMR features a new type of scoring wheel, harder and smaller than conventional scoring wheels, mounted in a self-adjusting cartridge which makes breaking even thick glass easy. The controlled, even pressure ensures the final, perfect break.

Leica's ultramicrotomy glass strips are made from a special type of plate glass which is superior to the "float" glass sometimes used for ultramicrotomy.

High quality cryoknives can be made routinely with two new scoring positions, enabling optimal comer to comer breaks. It simplifies the production of cryoknives with an angle as close to $45^{\circ}$ as possible. The LEICA EM KMR also features a glass-strip holder and locking devices for the adjustments, offering increased security of operation.

Knives can be produced in the traditional manner or by applying the "balanced break" concept. The balanced break technique enables you to start out with a standard $400 \mathrm{~mm}$ long strip of glass in any of three thickness, $6,4,8$, or $10 \mathrm{~mm}$ and continuously break the strip into equal halves. You will then have a full set of flat sided $25 \times 25 \mathrm{~mm}$ squares, perfect for making $45^{\circ}$ knives that measure up to your most stringent requirements

For further information regarding the LEICA EM KMR knifemaker contact Leica Inc, 111 Deer Lake Road, Deerfield, IL. 60015, Tel: (800)248-0123, Fax: (847) 405-0147. Circle Reader Inquiry \#42

\section{New TEM from Philips}

Philips Electron Optics recently announced a new transmission electron microscope, the Philips CM120 Biofilter. The instrument uses a post-column energy filter to achieve excellent image contrast in life science samples. Full analytical capability is achieved using Energy Dispersive $X$-ray analysis, Energy Filtered Imaging and Electron Energy Loss Spectroscopy (EELS).

The CM120 Biofilter has been optimized for ease of use and combines rotation-free imaging with a wide field of view. TV rate and slow scan CCD cameras are included for digital image recording, as is image processing software for contrast enhancement, elemental mapping and electron energy loss spectroscopy. Philips Electron Optics Low Dose software can now be used seamlessly with energy filtered image acquisition.

The CM120 BioFilter has all the benefits of the field-proven CM120 BioTWIN. These include an innovative objective lens that offers a high contrast image. The column geometry features a large collection angle for Energy Dispersive $X$-ray (EDX) systems. In addition, large cryo shields around the sample ensure a clean vacuum and provide many hours of contamination-free observation of both cooled and uncooled specimens. The instrument's CompuStage provides high stability and eucentricity, also at cryogenic temperatures

The main application areas for the instrument are imaging of thick samples in cell biology and cryo microscopy. More specialized applications of the instrument include electron crystallography and automated tomography in structural biology and in combined morphological and analytical studies in pharmaceutical and environmental research.

For further information, contact Philips Electronic Instruments: (201)529-6246, Fax: (201)529-5084. Circle Reader Inquiry \#44

\section{High Frame Rate Cooled CCD Camera}

The C4480-81/82 High Frame Rate Cooled CCD Cameras are newly designed for high frame rate measurements with sensitivity comparable to conventiona cooled CCD cameras. The C4880-81/82 incorporates a new progressive-scan interline $\mathrm{CCD}$ which requires no mechanical shutter, enabling vibration free measurement under microscopes.

The $04880-81 / 82$ has $656 \times 494$ pixels employing dual scan capability, the high speed mode gives approximately $500 \mathrm{~Hz}(32 \times 32$ binning, $28 \mathrm{~Hz}$ for full resolution) frame rate at typical 20 e read-out noise while the high precision mode gives approximately $320 \mathrm{~Hz}$ ( $32 \times 32$ binning, $1 \mathrm{~Hz}$ for full resolution) frame rate at typical 5 e read-out noise. The CCD utilizes peltier cooling, which allows maximum 60 seconds exposure time.

Hamamatsu Photonic Systems: (508)671-5000, Fax. (508)671-9415. Circle Reader Inquiry \#45

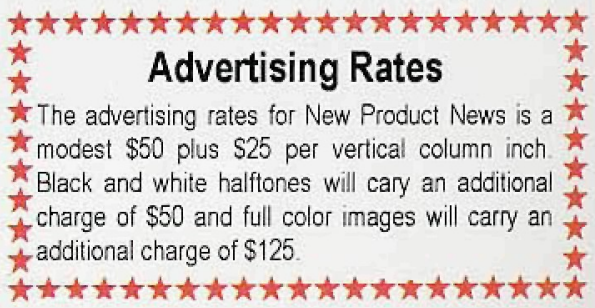

